\title{
Targeting Gene-Virotherapy of Cancer and its prosperity
}

\author{
Xin Yuan Liu ${ }^{1,2}$ \\ ${ }^{1}$ Institute of Biochemistry and Cell Biology, Shanghai Institutes for Biological Sciences, Chinese Academy of Sciences, 320 Yue \\ Yang Road, Shanghai 200031, China; ${ }^{2}$ Xinyuan Institute of Medicine and Biotechnology, School of Life Science, Zhejiang Sci-Tech \\ University, Hangzhou 310018, China
}

Gene and viral therapies for cancer have shown some therapeutic effects, but there has been a lack of real breakthrough. To achieve the goal of complete elimination of tumor xenograft in animal models, we have developed a new strategy called Targeting Gene-Virotherapy of Cancer, which aims to combine the advantages of both gene therapy and virotherapy. This new strategy has produced stronger anti-tumor effects than either gene or viral therapy alone. A tumorspecific replicative adenovirus vector, designated as ZD55, was constructed by deletion of the 55kDa E1B region of adenovirus. The resulting viral construct not only retains a similar function to ONYX-015 by specifically targeting $p 53$ negative tumors, but also allows for the insertion of various therapeutic genes to form appropriate ZD55 derivatives due to the newly introduced cloning site, a task not feasible with the original ONYX-015 virus. We showed that the anti-tumor effect of one such derivative, ZD55-IL-24, is at least 100 times more potent than that of either ZD55 virotherapy or Ad- $I L-24$ gene therapy. Nevertheless, complete elimination of tumor mass by the use of ZD55-IL-24 was only observed in some but not all mice, indicating that one therapeutic gene was not sufficient to "cure" these mice. When genes with complementary or synergetic effects were separately cloned into the ZD55 vector and used in combination (designated as the Dual Gene Therapy strategy), much better results were obtained; and it was possible to achieve complete elimination of all the xenograft tumor masses in all mice if two suitable genes were chosen. More comprehensive studies based on this new strategy will likely lead to a protocol for clinical trial. Finally, the concept of Double Controlled Targeting Virus-Dual Gene Therapy for cancer treatment, and the implication of the recent progress in cancer stem cells are also discussed.

Cell Research (2006) 16:879-886. doi:10.1038/sj.cr.7310108; published online 14 November 2006

Keywords: targeting therapy, cancer therapy, gene-virotherapy

\section{Introduction}

Gene therapy was first carried out in patient by Anderson in 1990 to treat a girl with SCID (Severe Combined Immunodeficiency Disease) that results from the deficiency of the $A D A$ gene. To date more than 1000 gene therapy protocols have been used in clinic, among which $63-67 \%$ were for cancer treatment. Nevertheless, significant breakthrough has been lacking in the field. Viral therapy was used about a hundred years ago when some cancer patients got remission after getting virus infection. In 1970, there

Correspondence: Xin Yuan Liu

Tel/Fax: 86-021-54921126, 86-0571-86843185

E-mail: xyliu@sibs.ac.cn were 38 different viruses being used in patients. Now many viruses - especially the modified viruses - are formally used in clinic, such as G207 derived from HSV-1, CV706 from Ad5, CV787 also from Ad5, HSV-1716, NV1020 from HSV-1/HSV-2, PV701 from NDV and vaccinia GM-CSF. The term virotherapy was suggested in 2001 by Kirn [1]. For instance, when used in combination with $5 \mathrm{FU}$ and cisplatin, the ONYX-015 led to a $63 \%$ anti-tumor effect in a phase II trial [2], but the anti-tumor efficacy was only 15-20\% when ONYX-015 was used alone [2]. ONYX-015 had passed phase II and entered phase III clinical trial, but the trial was eventually stopped. Generally, there has also been no big breakthrough in virotherapy.

The ability to specifically target cancer cells (Targeting) would be crucial for a successful cancer therapy. To this 


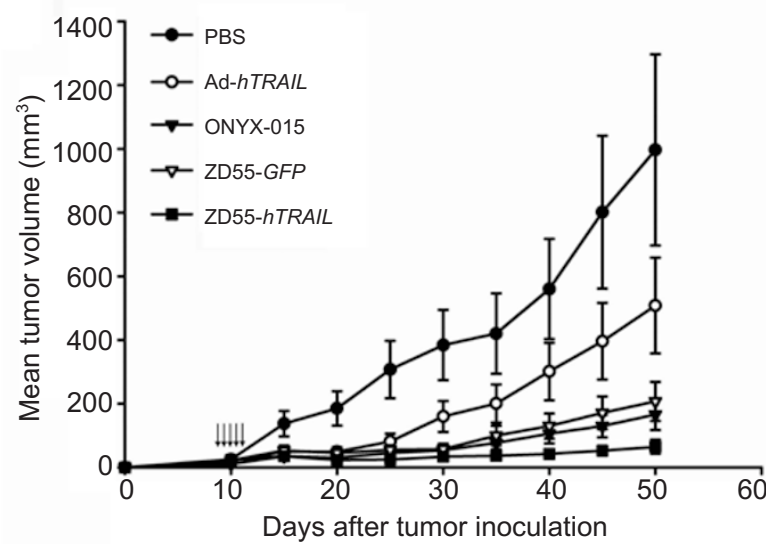

Figure 1 Enhanced anti-tumor effect of targeting gene-virotherapy (ZD55-hTRAIL) in vivo. Tumors were established in nude mice by implantation of SW620 cells. When tumor size reached $60-80 \mathrm{~mm} 3$, animals were treated by intratumor injection of PBS or Ad- $h T R A I L$, ZD55-GFP, ONYX-015, or ZD55- $h$ TRAIL at $2 \times 10^{8} \mathrm{pfu} /$ animal daily for 5 consecutive days. Tumor size was measured every 5 days and is presented as mean $\pm \mathrm{SD}(\mathrm{n}=6)$. The inhibitory effect of ZD55hTRAIL at the end of 50 days on tumor growth was better than that of virotherapy alone $(P<0.05$, versus ONYX-015 or ZD55-GFP) and control animals receiving PBS $(P<0.001)$. Adapted with permission from Mol Ther 2005; 11:531-541 [7].

end, we have initiated a strategy, designated as Targeting Gene-Virotherapy of Cancer, by taking the advantage of both gene therapy and virotherapy [3]. Our first targeting virus constructed was ZD55, which was derived by deleting the E1B 55kDa gene from adenovirus [4]. ZD55 is similar to ONYX-015 in its ability to selectively target and infect $p 53$ negative (or $p 53$ signaling pathway defective) cancers, but ZD55 was significantly different from ONYX-015 in many other features. In addition to differences in the construction method, our ZD55 was derived from Ad5 while ONYX-015 was from the fusion virus Ad2/Ad5; furthermore, ZD55 contained a cloning site designed to insert foreign gene(s), whereas no cloning site was available in ONYX-015. Our strategy of Targeting Gene-Virotherapy can be divided to three stages.

\section{Using one therapeutic gene}

The first stage of our strategy involves the use of one therapeutic gene (Targeting Gene-Virotheapy of Cancer, patent No. 02157662.9). We cloned many genes individually into ZD55 to form ZD55-gene(s) and examined the anti-cancer efficacy of the resulting ZD55-gene(s). A number of papers have been published from our group in recent years on different gene(s), such as CD [4], sFlt1 [13], (an inhibitor of VEGF consisting of the first three extra cellular domain of soluble Flt-1, the hVEGF receptor-1) [5], TRAIL (tumor-necrosis factor-related apoptosis-inducing ligand) [6], $K 5$ (kringle 5 of plasminogen) [7], Smac (Second mitochondria-derived activator of caspases) [8], XAF-1 (XIAP associated factor-1) [9], MnSOD [10], IL-24 [11], and hSSTr2 (somatostatin receptor gene subtype 2) [12], etc. We have recently expanded this strategy to include a gene coding for an siRNA against X-IAP (X-linked inhibitor of apoptosis) (submitted). The anti-tumor effects of all these ZD55-gene constructs are much better than the respective gene therapy or virotherapy alone as shown in Figure 1 for ZD55-TRAIL [7]. Another special case is ZD55-IL-24 (patent No. 2005 10026151.5). Its anti-tumor effect is about 100-fold more potent than ZD55 or ONYX015 , or the gene therapy product Ad- $I L-24$ [11] which has entered phase II clinical trial in USA [13]. We think that the following reasons may account for the success of our strategy. ZD55 is a replicative and targeting vector, which can amplify several hundred to several thousand times in the targeted tumor cells while its carried gene(s) can also simultaneously be amplified several hundred to several thousand times. These characteristics of ZD55-gene overcome the disadvantage of traditional gene therapy involving a replication-deficient adenovirus that results in lower expression of the therapeutic gene with no targeting effect, and also improve the killing effect of traditional virotherapy (such as ZD55 or ONYX-015). It is our prediction that a future trend of gene therapy or virotherapy for cancer will be the strategy of Targeting Gene-Virotherapy. We first proposed the term "Targeting Gene-Virotherapy", and our group in recent years made major efforts to extensively test this strategy; although there were a few previous reports on delivering therapeutic genes using oncolytic viruses, such work on ONYX-015 was restricted with the tk or cd gene only $[14,15]$.

\section{Using two therapeutic genes}

The second stage of our strategy involves the use of two anti-tumor genes (Targeting Dual Gene-Virotherapy of Cancer, patent No. 0210569.9). Although a lot of work has been done in our lab for ZD55-genes and in some cases the tumor mass can be completely eliminated in some mice, the use of any single ZD55-gene could never be able to eliminate all the tumors in all mice. Therefore we developed the Dual Gene strategy. We reason that the use two therapeutic genes together may have additive, complementary or synergetic effect, leading to a much stronger anti-tumor effect than using one gene alone. For example, K5 is a strong anti-angiogenesis factor and TRAIL is a very strong apoptotic factor. When ZD55-TRAIL and Ad-K5 and were used together, their complementary effect led to the complete elimination 


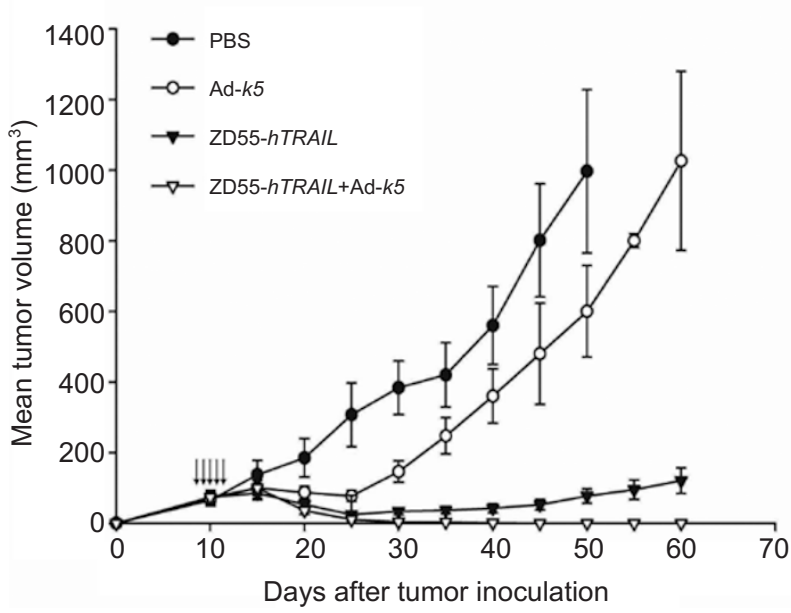

Figure 2 The superiority of targeting dual gene-virotherapy strategy. Anti-tumor effect of targeting dual gene-virotherapy by combination of ZD55-hTRAIL and Ad- $k 5$. When SW620 tumor size reached $70-100 \mathrm{~mm}^{3}$, animals were treated by intratumor injection of PBS or Ad- $k 5\left(2 \times 10^{8}\right.$ pfu daily) or ZD55- hTRAIL $\left(2 \times 10^{8}\right.$ pfu daily $)$ alone or a combination of ZD55- $h$ TRAIL $\left(1.8 \times 10^{8}\right.$ pfu daily) and Ad- $k 5$ ( 0 . $2 \times 10^{8}$ pfu daily) for 5 consecutive days. Tumor size was measured every 5 days and is presented as mean $\pm \operatorname{SD}(n=6)$. Adapted with permission from Mol Ther 2005; 11:531-541 [7].

of the SW620 colorectal tumor xenograft in all examined nude mice as shown in Figure 2 [7].

We have performed a number of studies using this strategy of Targeting Dual Gene-Virotherapy of Cancer. Our main results can be summarized as follows. The combination of ZD55-TRAIL and Ad-K5, or ZD55-TRAIL and ZD55-Mn$S O D$, or ZD55-TRAIL and ZD-IL-24, could completely eradicate all SW620 colorectal cancer xenograft in all nude mice [7, 10, 16]. ZD55-TRAIL + ZD55-Smac could completely eliminate all BEL 7404 hepatoma xenograft in all nude mice [8]. ZD55-TRAIL + ZD55-hSSTr 2 could completely eliminate all BxPC-3 pancreatic adenocarcinoma xenograft in nude mice [12]. AAV-hTERT-TRAIL + AAV-hTERT-IFN- $\beta$ could completely eliminate all A549 lung cancer xenograft in all nude mice (submitted).

Among the above reported cases, ZD55-TRAIL proves to be an important drug which can be combined with many other ZD55-genes to completely eradicate all the xenograft cancers. TRAIL is a good anti-tumor factor, as it shows no toxicity to liver when it is expressed in a targeting vector under the hTERT promoter [17] and it possesses the bystander effect [18] which is very important for the killing all tumor mass. Thus, it might be desirable to further test ZD55-TRAIL on a larger scale. We plan to conduct phase I/II clinical trials for ZD55-IL-24 in 2007 and for ZD55-TRAIL in 2008. It is possible that the combination
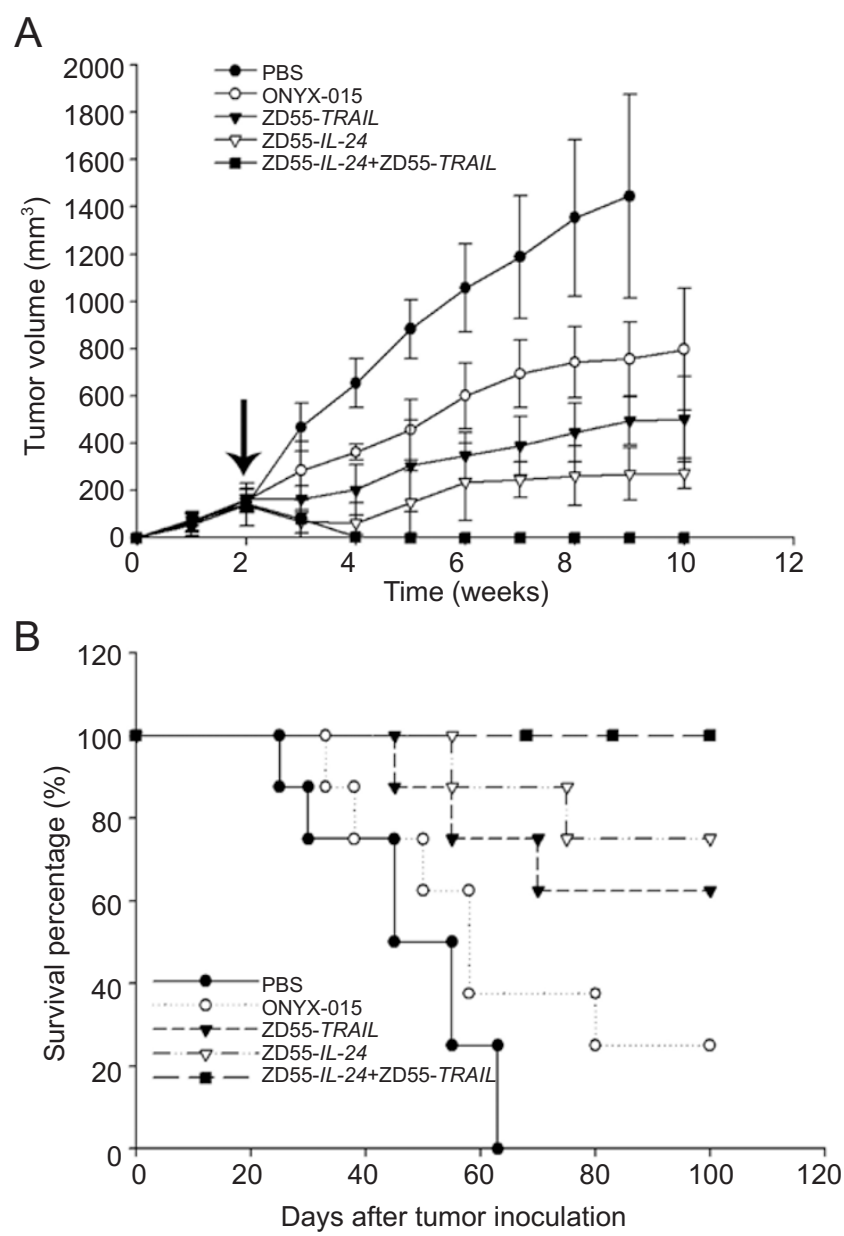

Figure 3 Complete eradication of human SW620 xenograft tumor in nude mice by the co-administration of ZD55-IL-24 and ZD55TRAIL. When tumor size reached $100-150 \mathrm{~mm}^{3}$, subcutaneous tumor-bearing mice were divided into four groups and treated with four consecutive daily intratumoral injections of PBS or with ZD55$I L-24, \mathrm{ZD} 55-T R A I L$ and the combination at $5 \times 10^{8} \mathrm{pfu} /$ dose per day (treatment indicated by arrow). (A) The tumor size was measured using calipers and tumor volume was calculated. Data are presented as means of tumor volume $\pm \mathrm{SD}$. $(\mathrm{n}=8)$. (B) The death of animals was monitored. Long-term survival of animals was observed after treatment with oncolytic adenoviruses compared with control animals receiving saline. Adapted with permission from Cancer Gene Ther 2006; 13:1011-1022 [16].

of ZD55-TRAIL and ZD55-IL-24 will lead to a very good protocol for the treatment of cancer patients, since together they could completely eliminate all the colorectal cancer xenograft as shown in Figure 3 [16]. The mechanism of their action was shown in Figure 4 [16]. It is our hope that the Targeting Dual Gene-Virotherapy strategy will produce excellent clinical therapeutical effect for patients in the future. However, it should be noted that delivering two genes in the replication-deficient vector under conventional 


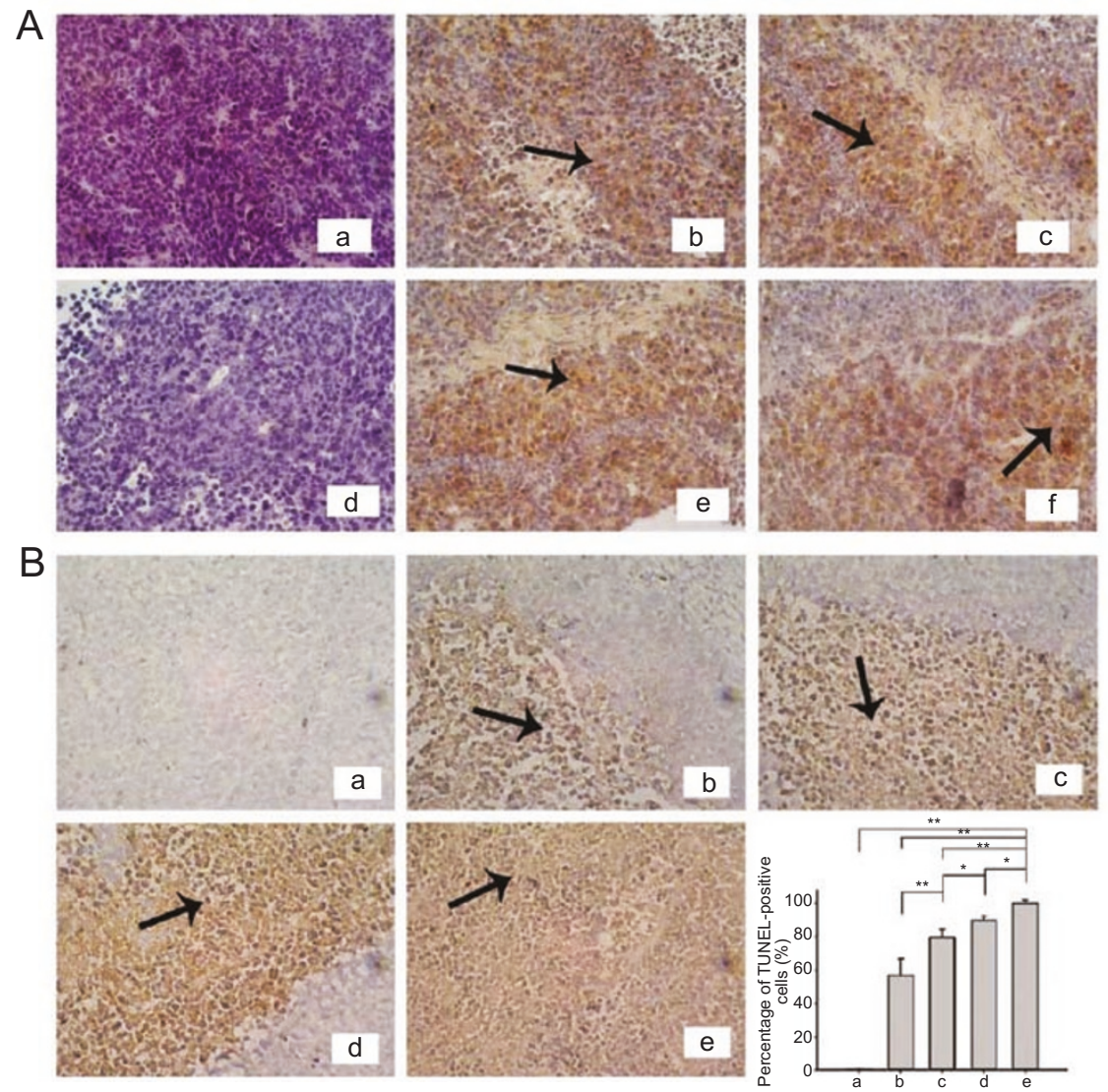

Figure 4 Immunohistochemical staining and TUNEL assay. (A) SW620 tumors subcutaneously receiving various treatments were harvested 3 days after infection and tumor sections were analyzed for the $I L-24$ (a-c) and TRAIL (d-f) expression by immunohistochemistry (400 original magnification). The stained signals are indicated with a brownish black arrow, and no expression of $I L-24$ or TRAIL was seen in mock infection $(\mathrm{a}, \mathrm{d})$. The $I L-24$ expression in the tumor sections was observed in the case of the tumors injected with ZD55-IL-24 (b) or with ZD55-IL-24 and ZD55-TRAIL (c). Similar results were also obtained for the detection of TRAIL expression (e), ZD55-TRAIL infection; (f), ZD55-TRAIL and ZD55-IL-24 infection. (B) TUNEL assay was also performed to detect apoptosis in the sections of tumors that received different treatments. (a) tumors treated with PBS as control; (b) tumors treated with ONYX-015; (c) Tumors treated with ZD55-TRAIL; (d) tumors treated with ZD55-IL-24; (e) tumors treated with ZD55-IL-24 and ZD55-TRAIL. Arrows denote cells undergoing apoptosis. Percentage of TUNEL-positive cells to all cells is shown for each of the indicated treatments. Data are presented as mean \pm SD. $\left({ }^{*} P<0.05 ; * * P<0.01\right)$. Adapted with permission from Cancer Gene Ther 2006; 13:1011-1022 [16].

gene therapy was rarely found to completely eliminate all the xenograft tumor mass in all nude mice.

Two therapeutic genes can also be linked together and be cloned into the same viral vector, but there are several potential issues with this approach. First, it is not yet clear whether our SFDA would approve this kind of product with two linked genes for clinical use. The second issue is how to select the appropriate linker to join the two genes into the same vector. IRES and (glyglyglyglyser) 3 are considered out of date for use as the linker. For example, the expression level of gene $B$ in the construct of $A$-IRES- $B$ is usually 5-10 times lower than that of gene $A$. We are currently exploring the possibility of harnessing the self-cleavage property of the $2 \mathrm{~A}$ peptide from FMDV or using the four residue IETD motif as the TRAIL linker. For example, a TRAIL-IETD-Smac fusion will be specifically cleaved at $\mathrm{D} \downarrow$ of the IEPD motif by caspase 8 whose activity can be induced by TRAIL itself. Our animal studies have already shown that two anti-tumor genes delivered in the targeting vector (such as ZD55-gene 1 and ZD55-gene 2) are usually sufficient to kill all the tumor mass if the appropriate genes with complementary or synergetic effect were chosen. Given this, it seems not necessary to pursue the combinatorial use of three genes as it would be practically much more difficult and/or costly to achieve. 

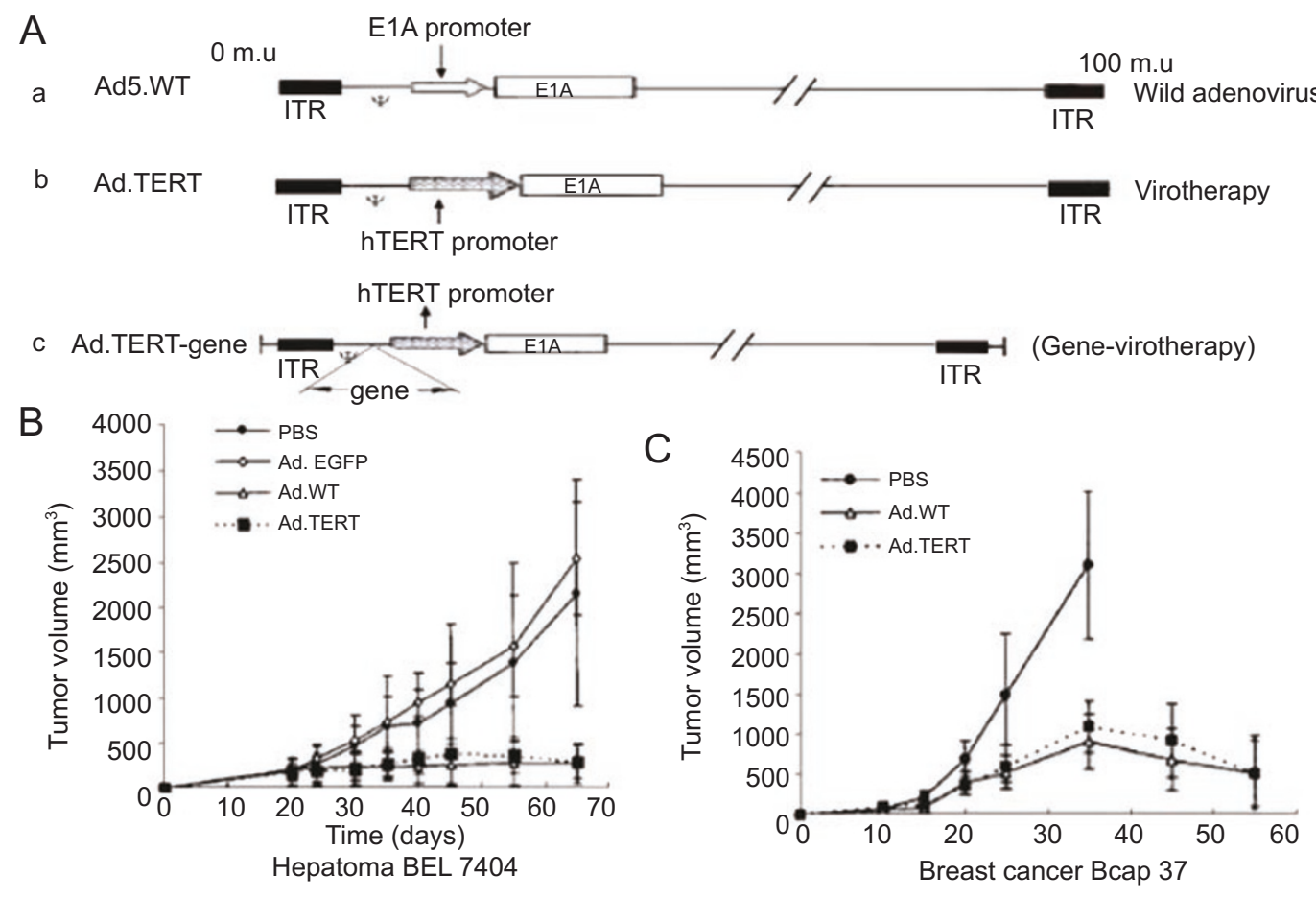

Figure 5 (A) Construction of Ad-hTERT-Gene for anti-tumor therapy. (a) In wild type Adenovirus, E1A is controlled by its own promoter. (b) hTERT promoter was used to replace the E1A promoter to form Ad-hTERT. (c) An anti-tumor gene (such as Trail) expression cassette was inserted into Ad-hTERT to form Ad-hTERT-gene. (B) Anti-tumor effect of Ad-hTERT for Hepatoma BEL 7404. (C) Anti-tumor effect of Ad-hTERT for Breast cancer Bcap 37. Adapted with permission of Ai Zheng 2006; 23:385-392 [26].

\section{Using two targeting promoters and two therapeutic genes}

The third stage of our strategy involves the use of two targeting promoters and two anti-tumor genes, and we designate it as Double Controlled Targeting Virus-Dual Gene Therapy of Cancer. In this case, the eventual tumortargeting oncolytic viruses were constructed by the use of two different tumor-specific promoters to control the essential viral genes, thus resulting in greater specificity against the targeted tumors.

For example, the use of hTERT (human telomerase reverse transcriptase, which is up-regulated in about $90 \%$ of tumors) promoter to control adenovirus E1A results in Ad-hTERT, which could target a broad range of cancers such as hepatoma or breast cancers (Figure 5) as shown in our previous paper $[19,26]$; this is in contrast to the PSA promoter which can only target prostate cancer. The Ad-hTERT is nevertheless still a targeting virotherapy reagent. After insertion of an anti-tumor gene as TRAIL to form Ad-hTERT-TRAIL, it became Targeting Gene-Virotherapy with a more potent anti-tumor effect [20-25]. To take advantage of features of both the Ad-hTERT vector and the ZD55-vector, a hybrid vector consisting of AdhTERT-E1A-ZD55 (E1B) was constructed and was named as TD55. Cloning of the TRAIL gene into TD55 generated TD55-TRAIL. Interestingly, both TD55 and TD55-TRAIL demonstrated better safety for normal cells such as MRC5 and WI38 (Figure 6); and TD55-TRAIL showed good antitumor effect against SW620 or A549 tumor cells (Figure 6) [26]. TD55-TRAIL can be considered as a vector for Double Controlled Targeting Virus-Gene Therapy, as it delivers a therapeutic gene (TRAIL) under a double-controlled virus (E1A is controlled by the hTERT promoter, and the deletion of E1B offers another control).

After consideration of all the above data, we are interested in further developing a new strategy, Double Controlled Targeting Virus-Dual Gene Therapy, for the treatment of individual cancers. Some specific protocols will be designed. There are three crucial points that need to be considered, taking the hepatoma as an example. First, there should be double cancer-specific promoters to control the viral replication. For example, the E1A of adenovirus can be controlled by the hTERT promoter and the E1B 

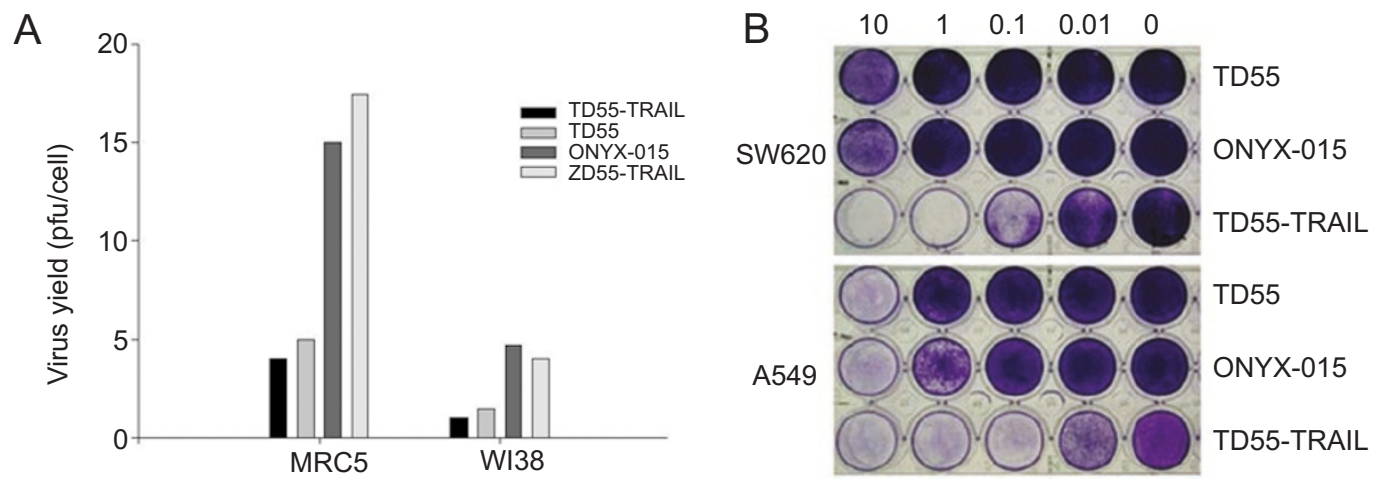

Figure 6 (A) Replication of TD55 and TD55-TRAIL in MRC5 and WI38 cells. (B) Selective effects of TD55 and TD55-TRAIL on SW620 and A549 cells, Adapted with permission of Ai Zheng 2006; 23:385-392 [26].

can be controlled by the AFP ( $\alpha$-fetoprotein) promoter, a promoter specific for liver cancer. The resulting adenovirus would be a double tumor promoter controlled vector of Ad5-hTERT-E1A-AFP-E1B which is expected to have higher cancer specificity. Second, the expression of a specific cancer suppressor gene, for example, the liver cancer suppressor gene HCCS1 (or LFIRE), can be controlled by the AFP promoter to generate a construct such as AdhTERT-E1A-AFP-E1B-HCCS1 (or LFIRE). In a preliminary study ZD55-HCCS1 has shown a very good anti-tumor effect (data not shown). It is a specific and individualized therapy for hepatocellur cancer (HCC) by using two cancer specific promoters, with one HCC specific promoter AFP to direct the expression of a hepatoma suppressor gene (HCCS1 or LFIRE). We speculate that constructs like AdhTERT-E1A-AFP-E1B-HCCS1 will also have very good anti-tumor effect. Third, if constructs such as Ad-hTERTE1A-AFP-E1B-HCCS1 (or LFIRE) could not completely eliminate all the hepatoma xenograft, we will generate additional constructs with predicted good anti-tumor effect such as Ad-hTERT-E1A-AFP-E1B-IL-24. The combined use of Ad-hTERT-E1A-AFP-E1B-HCCS1 (or LFIRE) and Ad-hTERT-E1A-AFP-E1B-IL-24 would realize the idea of Dual Gene therapy strategy as discussed above; and it is predicted that all the BEL-7404 hepatoma xenograft would be completely eliminated by this combination approach. Similarly, the colorectal cancer could be treated by the use of colorectal cancer specific suppressor gene such as $S T 13$ and the gastroenterological promoter CEA. All the above protocols are currently being studied. The same principle can be also applied to lung cancer, prostate cancer etc. The safety of a double controlled targeting vector has been confirmed (Figure 6) [26]. In the above discussion I have outlined the general principle for conducting Double Controlled Targeting Virus-Dual Gene Therapy of Cancer. It is conceivable that changes and modifications can be made to meet the needs of specific procedures. For the example, the alternative choice for the targeting control promoter is the surviving promoter (SurP) or the hTERT-E1A $(\Delta 24)$, both of which were better than hTERT $[27,28]$; and for the killer gene, one can use TRAIL, MnSOD or others instead of $I L-24$. In summary, it is expected that the strategy of Double Controlled Targeting Virus-Dual Gene Therapy of Cancer could be used to design many excellent protocols to achieve ideal therapeutic effect for many individual cancers, and we hope that its successful implementation will lead to a cure for at least some cancers in the not far future.

Finally, it is worthwhile to consider our strategy in the context of the recent progress in cancer therapy. There are three critical issues in cancer therapy. First, early detection remains a key, as reflected by Laura Spinney's recent writing in Nature [29] (August 17, 2006), "The detection of cancer at an early stage in its development can be life saving". Second, cancer stem cell is the cause of tumor recurrence; therefore it is a root problem for cancer therapy to kill the cancer stem cell. Many cancer stem cells have been discovered including those in leukemia [30], breast cancer [31], brain tumor [32], and prostate cancer [33, 34]; and these have been extensively reviewed elsewhere [35-38]. Third, targeting therapy of cancer is another key. To develop drugs that target and kill cancer cells without impairing the normal cells is an important problem in cancer therapy. Recently, a Xiangshan conference (similar to Gorden conference in USA) on Targeting Therapy of Cancer was held (March 22-24, 2005) in China [39]. There are a number of directions and approaches to target cancer cells including, for example: 1), antibody therapy (24 antibodies have been licenced to market among which 8 are anti-tumor antibodies such as Herceptin and Avastin); 2), antibody gene therapy [40, 41] which offers an attractive alternative to the traditional antibody therapy [42]; 3), targeting gene-virotherapy, which can completely 
eliminate xenograft tumors in nude mice by the use of two appropriate therapeutic genes [7]; 4), targeting cancer stem cells; 5), targeting an intracellular protein tyrosine kinase (such as Gleesvec, which was selected as one of the 10 greatest science contributions in 2001); and 6), targeting the EGFR Kinase [43]. It is of no doubt that progress in our ability to target cancer will continue to contribute to better cancer therapy.

\section{Acknowledgment}

The author thanks Prof Guofan Hong and Associate Prof Jin-fa Gu for critical review of my manuscript and for reading the manuscript. This work was supported by grants from Hi-Tech Research and Development Program of China (863 program) (No. 2003 AA216031 and No. 2002 AA216021) and National Basic Research Program of China (973 Program) (No. 2004 CB518804).

\section{References}

1 Kirn D, Martuza RL, Zwiebel J. Replication-selective virotherapy for cancer: Biological principles, risk management and future directions. Nat Med 2001; 7:781-787.

2 Khuri FR, Nemunaitis J, Ganly I, et al. a controlled trial of intratumoral ONYX-015, a selectively-replicating adenovirus, in combination with cisplatin and 5-fluorouracil in patients with recurrent head and neck cancer. Nat Med 2000; 6:879-885.

3 Liu XY. A new anticancer strategy-genetic and virological treatment of cancer. Chin J Cancer Biother 2001; 8:1.

4 Zhang ZL, Zou WG, Luo CX, et al. An armed oncolytic adenovirus system, ZD55-gene, demonstrating potent anti-tumoral efficacy. Cell Res 2003; 13: 481-489.

5 Zhang Z, Zou W, Wang J, et al. Suppression of tumor growth by oncolytic adenovirus-mediated delivery of an antiangiogenic gene, soluble Flt-1. Mol Ther 2005; 11:553-562.

6 Qiu S, Ruan H, Pei Z, et al. Combination of Targeting Gene-ViroTherapy with 5-FU enhances anti-tumor efficacy in malignant colorectal carcinoma. J Interferon Cytokine Res 2004; 24:219230.

7 Liu XY, Qiu SB, Zou WG, et al. Effective gene-virotherapy for complete eradication of tumor mediated by the combination of hTRAIL (TNFSF10) and plasminogen k5. Mol Ther 2005; 11:531-541.

8 Pei Z, Chu L, Zou W, et al. An oncolytic adenoviral vector of Smac increases anti-tumor activity of TRAIL against HCC in human cells and in mice. Hepatology 2004; 39:1371-1381.

9 Qi R, Liu XY. New advance in caspase-independent programmed cell death and its potential in cancer therapy. Cancer Gene Ther, in press.

10 Zhang Y, Gu J, Zhao L, et al. Complete elimination of colorectal tumor xenograft by combined manganese superoxide dismutase with tumor necrosis factor-related apoptosis-inducing ligand gene virotherapy. Cancer Res 2006; 66:4291-4298.

11 Zhao L, Gu J, Dong A, et al. Potent anti-tumor activity of oncolytic adenovirus expressing mda-7/IL-24 for colorectal cancer.
Hum Gene Ther 2005; 16:845-858.

12 Zhang Z, Gu J, Dong A, et al. Dual-purpose oncolytic adenovirus bearing human somatostatin receptor subtype 2 gene: Imaging gene transfer and enhancing TRAIL-induced anti-tumor efficacy. Cancer Res, in press.

13 Fish P. Is mda-7/IL-24 a "magic bullet" for cancer? Cancer Res 2005; 65:10128-10138.

14 Nanda D, Vogels R, Havenga M, Avezaat CJ, Bout A, Smitt PS. Treatment of malignant gliomas with a replicating adenoviral vector expressing herpes simplex virus-thymidine kinase. Cancer Res 2001; 61:8743-8750.

15 Freytag SO, Rogulski KR, Paielli DL, Gilbert JD, Kim JH. A novel three-pronged approach to kill cancer cells selectively: concomitant viral, double suicide gene, and radiotherapy. Hum Gene Ther 1998; 9:1323-1333.

16 Zhao L, Dong A, Gu J, et al. The anti-tumor activity of TRAIL and IL-24 with replicating oncolytic adenovirus in colorectal cancer. Cancer Gene Therapy 2006; 13:1011-1022.

17 Lin T, Gu J, Zhang L, et al. Targeted expression of green fluorescent protein/tumor necrosis factor-related apoptosis-inducing ligand fusion protein from human telomerase reverse transcriptase promoter elicits anti-tumor activity without toxic effects on primary human hepatocytes. Cancer Res 2002; 62:3620-3625.

18 Kagawa S, He C, Gu J, et al. Anti-tumor activity and bystander effects of the tumor necrosis factor-related apoptosis-inducing ligand (TRAIL) gene. Cancer Res 2001; 61:3330-3338.

19 Zou W, Luo C, Zhang Z, et al. A novel oncolytic adenovirus targeting to telomerase activity in tumor cells with potent. Oncogene 2004; 23:457-464.

20 Tang Y, Chen Y, Gu J, et al. Killing effect of Ad.TERT-TRAIL on tumor cell lines and its mechanism. Ai Zheng 2005; 24:536542.

21 Zhang Q, Nie M, Sham J, et al. Effective gene-viral therapy for telomerase-positive cancers by selective replicative-competent adenovirus combining with endostatin gene. Cancer Res 2004; 64:5390-5397.

$22 \mathrm{Gu}$ J, Kagawa S, Takakura M, et al. Tumor-specific transgene expression from the human telomerase reverse transcriptase promoter enables targeting of the therapeutic effects of the Bax gene to cancers. Cancer Res 2000; 60:5359-5364.

$23 \mathrm{Gu}$ J, Andreeff M, Roth JA, et al. hTERT promoter induces tumor-specific Bax gene expression and cell killing in syngenic mouse tumor model and prevents systemic toxicity. Gene Ther 2002; 9:30-37.

24 Koga S, Hirohata S, Kondo Y, et al. A novel telomerase-specific gene therapy: gene transfer of caspase- 8 utilizing the human telomerase catalytic subunit gene promoter. Hum Gene Ther 2000; 11:1397-1406.

25 Majumdar AS, Hughes DE, Lichtsteiner SP, et al. The telomerase reverse transcriptase promoter drives efficacious tumor suicide gene therapy while preventing hepatotoxicity encountered with constitutive promoters. Gene Ther 2001; 8:568-578.

26 Wang J, Gu JF, Yang SY, et al. Security of dual cancer-specific targeting vectors and its cytotoxic effect when harbored. Ai Zheng 2006; 25:385-392.

27 Kamizono J, Nagano S, Murofushi Y, et al. Survivin-responsive conditionally replicating adenovirus exhibits cancer-specific and efficient viral replication. Cancer Res 2005; 65:5284-5291.

28 Cai R, Zhang W, Dong A, et al. The oncolytic adenovirus target- 
ing to TERT and Rb pathway induced specific cytotoxicity to tumor cells. Mol Ther 2006; 13: S55.

29 Spinney L. Cancer: caught in time. Nature 2006; 442:736-738.

30 Bonnet D, Dick JE. Human acute myeloid leukemia is organized as a hierarchy that originates from a primitive hematopoietic cell. Nat Med 1997; 3:730-737.

31 Al-Hajj M, Wicha MS, Benito-Hernandez A, et al. Prospective identification of tumorigenic breast cancer cells. Proc Natl Acad Sci U S A 2003; 100:3983-3988.

32 Singh SK, Hawkins C, Clarke ID, et al. Identification of human brain tumor initiating cells. Nature 2004; 432:396-401.

33 Collins AT, Berry PA, Hyde C, et al. Prospecitive identification of tumorigenic prostate cancer stem cells. Cancer Res 2005; 65:10946-10951.

34 Wang S, Garcia AJ, Wu M, et al. Pten deletion leads to the expansion of a prostatic stem/progenitor cell subpopulation and tumor initiation. Proc Natl Acad Sci U S A 2006; 103:1480-1485.

35 Reya T, Morrison SJ, Clarke MF, et al. Stem cell, cancer, and cancer stem cells. Nature 2001; 414:105-111.
36 Pardal R, Clarke MF, Morrison S. Applying the principles of stem-cell biology to cancer. Nat Rev Cancer 2003; 3:895-902.

37 Abbott A. Cancer: the root of the problem. Nature 2006; 442:742743.

38 Passegue E. Cancer biology: a game of subversion. Nature 2006; 442:754-755.

39 Chu L, Liu XY. New trends of targeting cancer therapy. Chin J Cancer Prev Treat 2005; 10:1583-1588.

40 Guo MG, Jiang MH, Yang Q, et al. Gene therapy for ovarian cancers by adenovirus-mediated complete antibody gene. Zhonghua Yi Xue Za Zhi 2004; 84:1147-1151.

41 Fang J, Qian JJ, Yi S, et al. Stable antibody expression at therapeutic levels using the 2A peptide. Nat Biotechnol 2005; 23:584-590.

42 Liu XY. Prospects of antibody therapy and antibody gene therapy of carcinoma. Zhonghua Yi Xue Za Zhi 2004; 84:1145-1146.

43 Ji H, Li D, Chen L, et al. The impact of human EGFR kinase domain mutations on lung tumorigenesis and in vivo sensitivity to EGFR targeted therapies. Cancer Cell 2006; 9:485-495. 